\title{
Necropsy findings in lysinuric protein intolerance
}

\author{
D T McManus, R Moore, C M Hill, C Rodgers, D J Carson, A H G Love
}

\begin{abstract}
Lysinuric protein intolerance (LPI) is a rare autosomal recessive inborn error of metabolism, characterised by defective transport of the cationic amino acids lysine, arginine and ornithine. To date there are few reported necropsy cases. This report describes the necropsy findings in a 21 year old female patient originally diagnosed as having LPI in 1973. Liver function tests deteriorated and immediately before death jaundice, hyperammonaemia, coma, metabolic acidosis, and a severe bleeding diathesis developed. At necropsy, there was micronodular cirrhosis of the liver with extensive fatty change in hepatocytes. The lungs showed pulmonary alveolar proteinosis. Immunofluorescence and electron microscopy revealed the presence of a glomerulonephritis with predominant IgA deposition. These necropsy findings reflect the spectrum of lesions reported in LPI, providing further evidence of an association between this condition and pulmonary alveolar proteinosis, cirrhosis and glomerulonephritis.
\end{abstract}

(f Clin Pathol 1996;49:345-347)

Keywords: lysinuric protein intolerance, necropsy findings, glomerulonephritis.

Lysinuric protein intolerance (LPI) is a rare autosomal recessive condition originally described in Finland. Approximately 100 cases have been reported to date. ${ }^{12}$ LPI is characterised by defective transport of the cationic amino acids lysine, arginine and ornithine across cell membranes. Defective absorption of these amino acids in the small intestine accompanied by impaired tubular reabsorption in the kidney reduces serum concentrations and increases urinary excretion. Uptake of these amino acids into hepatocytes is also reduced. The liver becomes deficient in ornithine and this inhibits the urea cycle. Patients with LPI experience intermittent postprandial hyperammonaemia and a spontaneous aversion to protein frequently develops. The presenting symptoms include recurrent vomiting and failure to thrive in infancy. Those who survive may develop hepatosplenomegaly.

Several candidate genes for LPI have been identified recently. ${ }^{1}$ This may ultimately lead to DNA testing for this condition. However, the complexity and diversity of cationic amino acid transporter proteins and transport regu- latory proteins suggest that the LPI phenotype may be heterogenous as a result of different types of mutations affecting more than one gene. ${ }^{1}$

\section{Case report}

This female patient presented originally at eight months with persistent vomiting and failure to thrive. ${ }^{3}$ At that time there was a marked increase in urinary lysine excretion and to a lesser extent ornithine and arginine. The urinary orotic acid concentration was also raised and casein protein loading tests increased the concentrations of all plasma amino acids except lysine, onithine and arginine. Serum lactate dehydrogenase activities were also raised. Lysinuric protein intolerance was diagnosed. A protein restricted diet was recommended and supplements of lysine, arginine and citrulline were prescribed. During the teenage years compliance with the diet and amino acid supplements was poor. Osteoporosis was diagnosed at the age of 19 years and calcium supplements were introduced.

A gradual deterioration in the patient's condition began in 1992, with episodic disturbances of liver function and hyperammonaemia. From late 1993, liver function tests progressively deteriorated and she was admitted to hospital in August 1994. Immediately before death, the patient became comatose, had persistently raised serum ammonia concentrations, metabolic acidosis and a coagulopathy. Despite intensive therapy, including intravenous arginine for the hyperammonaemia, the patient died. Consent for a necropsy examination was obtained.

\section{Necropsy findings}

The body was that of a well nourished female and was mildly jaundiced. Multiple petechiae were present over the neck and shoulders.

There was enlargement of the liver $(1650 \mathrm{~g})$, with widespread macro- and microvesicular steatosis within hepatocytes. There was a micronodular cirrhosis and cholestasis with proliferation of bile ductules (fig 1). The spleen was enlarged and congested; there was over a litre of ascites and early oesophageal varices were present in keeping with portal hypertension. The lungs were heavy (right $712 \mathrm{~g}$ and left $560 \mathrm{~g}$ ), with mild pleural thickening and on sectioning, they were airless and oedematous. On histological examination, there was a foamy pink exudate within the alveolar spaces that stained with periodic acid Schiff 


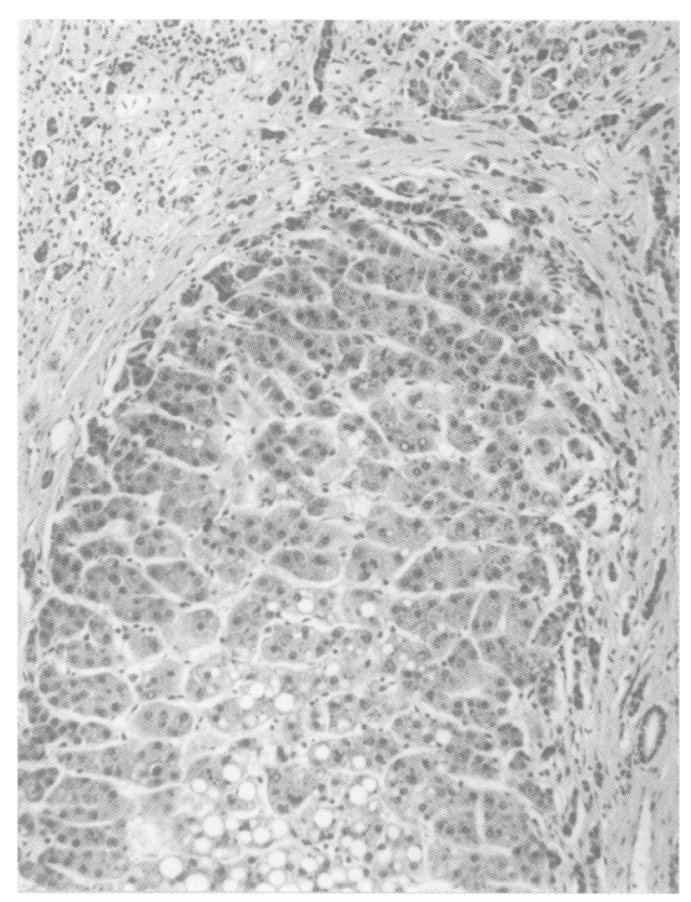

Figure 1 Fatty change in the cirrhotic liver. (Haematoxylin and eosin, $\times 200$.)

(PAS) diastase. There was no evidence of opportunistic infection.

The kidneys each weighed $160 \mathrm{~g}$. On sectioning, there was pallor and dystrophic calcification of the tips of the papillae. Histologically, there were patchy areas of tubular necrosis and tubular microcalcification. The glomeruli were mostly of normal cellularity with a few segments of mesangial hypercellularity in some. Crescents were not present. PAS staining showed focal thickening of glomerular basement membranes and staining with silver haematoxylin and eosin revealed occasional double contour glomerular capillary walls and a few that bore positive silver spikes. Direct immunofluorescence of snap frozen tissue showed positive staining for all immunoglobulins, particularly IgA, which was present in glomerular capillary walls and was also strongly positive in the mesangium (fig $2 \mathrm{~A}$ ). IgA staining was not seen in blood vessel walls. $\mathrm{C}^{\prime} 1 \mathrm{q}$ and $\mathrm{C}^{\prime} 3$ were also demonstrated in glomerular capillary walls. Ultrastructural examination of glutaraldehyde fixed, resin embedded tissue showed subepithelial, subendothelial and mesangial electron dense deposits, with foci of mesangial interposition (fig 2B).

Congo red staining showed no evidence of amyloid deposition within the spleen or lymph nodes. No abnormalities of the pancreatic acini or ducts were identified. Histology of the vertebrae confirmed osteoporosis and the bone marrow was hypercellular and reactive.

\section{Discussion}

The terminal illness of this patient with rapidly progressive multiple organ failure associated with a coagulopathy is very similar to that
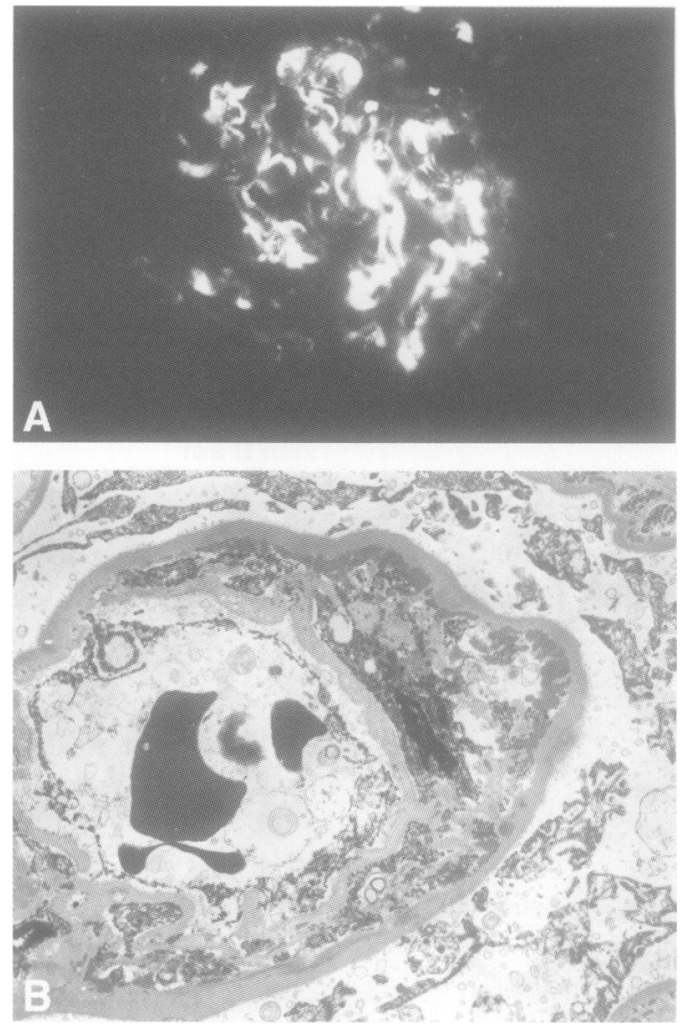

Figure 2 (A) Strong positive staining for $\operatorname{Ig} A$ in glomerular capillary walls and in the mesangium. (Direct immunofluorescence, $I g A \times 800$.) (B) Subendothelial deposits and splitting of the glomerular basement membrane with mesangial interposition. (Uranyl acetate, $\times$ 8000.)

recently described in three of four Finnish patients with LPI. ${ }^{2}$

The pulmonary haemorrhage and alveolar proteinosis in the current case were also present in the previous necropsy reports. There was no evidence of cholesterol granuloma formation or of opportunistic infection in the present case.

This patient survived until the age of 21 years, considerably older than the four Finnish patients, whose ages at death ranged from $3 \cdot 7$ to 13.3 years. While there was fatty change of the liver in all four previously reported cases, only one showed a micronodular cirrhosis. As this occurred in the youngest patient it would seem that factors other than the duration of the illness must be important. Protein malnutrition can produce fatty change in the liver because of reduced apolipoprotein synthesis. Administration of orotic acid can also induce fatty liver in experimental models by interfering with lipoprotein secretion ${ }^{4}$; orotic aciduria is a feature of LPI.

All four patients in the Finnish series had evidence of immune complex mediated glomerulopathy and there was evidence of impaired renal function in three. The histological findings and the distribution of the immune complexes bore some similarities to systemic lupus erythematosus (SLE) and in fact one patient had a positive antinuclear factor. A previous case report has documented antinuclear antibodies in LPI, although it is acknowledged that this could well be coincidental. ${ }^{5}$ The multiple locations of the de- 
posits and the "full" house immunofluorescence in our case do show some similarities to lupus nephritis. However, clinical examination did not suggest coexistent SLE; antinuclear antibodies were not measured.

There was also particularly strong staining for IgA, which was only described in one of the Finnish patients. Schönlein Henoch purpura is associated with IgA deposition and could produce this type of renal lesion. It is notable that a petechial rash was noticed terminally in our patient and that there were multiple haemorrhages over the serosal aspect of the bowel, but these could be secondary to the coexistent coagulopathy.

The liver plays a central role in clearance of immune complexes from the circulation and there is a well documented association between liver cirrhosis and a glomerulopathy characterised particularly by mesangial immune complexes and IgA deposition. ${ }^{6}$ The features described in this glomerulopathy are very sim- ilar to those seen in the current case; it may be that the glomerulopathy in LPI is related to concomitant liver injury rather than representing a distinctive lesion.

The authors wish to thank Mr J Sandford, Mr Roy Creighton and $\mathrm{Mr}$ Thomas McLoughlin for technical assistance.

Originally presented as a poster presentation at the 170th Meeting of The Pathological Society of Great Britain and Ireland.

1 Simell O. Chapter 18. Lysinuric protein intolerance and other cationic amino-acidurias. In: Scriver CR, ed. Lysinuric protein intolerance and other cationic aminoacidurias. The metabolic and molecular basis of inherited disease. New York: McGraw Hill, 1995:3603-27.

2 Parto K, Kallajoki M, Aho H, Simell O. Pulmonary alveolar proteinosis and glomerulonephritis in lysinuric protein intolerance: case reports and autopsy findings of four paediatric patients. Hum Pathol 1994;25:400-7.

3 Carson NAJ, Redmond OAB. Lysinuric protein intolerance. Ann Clin Biochem 1977;14:135.

4 Cotran RS, Kumar V, Robbins SL. Chapter 1. In: Cellular injury and adaptation. Pathologic basis of disease. 4th edn. Philadelphia: WB Saunders, 1989:21.

5 Nagata M, Suzuki M, Kawamura G, Kono N, Yamaguchi $\mathrm{S}$, Aoki K. Immunological abnormalities in a patient with lysinuric protein intolerance. Eur F Pediatr 1987;146:427-8. 6 Emancipator SN, Heptinstall RH. Chapter 6. In: IgA nephritis and Schonlein Henoch syndrome. Pathology of the kidney. 4th edn. Boston: Little Brown, 1992:443-51.

\title{
Intimal sarcoma of the right brachiocephalic vein presenting as the superior vena caval syndrome
}

\author{
E R C Rytina, Y K Govil, K Sabanathan, R Y Ball
}

\section{Departments of Histopathologyl Cytopathology, Norfolk \& Norwich Health Care NHS Trust, Brunswick Road, Norwich NR1 3SR E R C Rytina R Y Ball}

Medicine for the Elderly

Y K Govil

K Sabanathan

Correspondence to: Dr R Y Ball.

Accepted for publication 11 September 1995

\begin{abstract}
A case of an 84 year old man presenting with obstruction of the superior vena cava caused by an intimal sarcoma of the right brachiocephalic vein is reported. The tumour morphology was similar to intimal sarcomas arising in major arteries, a more common primary site, and showed malignant fibrous histiocytoma-like features. Immunohistochemistry was suggestive of myofibroblastic differentiation.

(f Clin Pathol 1996;49:347-349)
\end{abstract}

Keywords: intimal sarcoma, vein, superior vena caval syndrome.

Primary sarcomas of the great vessels are rare and usually arise in the aorta, inferior vena cava, or pulmonary artery. ${ }^{1}$ Sarcomas of the thoracic veins are of exceptional rarity and may be associated with the superior vena caval syndrome. ${ }^{12}$ Here, we describe a primary intimal sarcoma of the right brachiocephalic vein which presented in this manner.

\section{Case report}

An 84 year old man complained of increasing breathlessness, occasional bloodstaining of sputum, and worsening headache of one month's duration. He had had a cough, productive of mucoid sputum, for two years. The past medical history included: appendicitis; partial gastrectomy for perforated duodenal ulcer; emphysema; cervical spondylosis; prostatic resection for nodular hyperplasia; and inguinal herniorrhaphy. $\mathrm{He}$ was alert, orientated and thin, seemed to have lost weight, and showed central cyanosis, suffusion of the face, and conjunctival congestion and oedema. The neck veins were engorged to the mandibular angle and showed no pulsation. Those over the chest were dilatated and their flow was towards the abdomen. There was bronchial breathing with increased vocal resonance and some crepitations in the right infraclavicular region. Small hard mobile lymph nodes were palpable in both axillae. No other significant findings were made on clinical examination.

Chest $x$ ray showed emphysematous lung fields, possible enlargement of the right paratracheal lymph nodes and no evidence of mediastinal masses or lung tumours. A computed tomography scan of the neck and chest (fig 1) showed a mass filling the lumen of the right brachiocephalic and internal jugular veins with evidence of obstruction of the left brachiocephalic vein and superior vena cava. 